\title{
DPC Based Health Service Planning for Cancer Medicine
}

\author{
Shinya Matsuda ${ }^{1)}$, Koichi Benjamin Ishikawa2), Kazuaki Kuwabara ${ }^{3)}$, \\ Kenji Fujimori ${ }^{4)}$, Kiyohide Fushimi ${ }^{5)}$, Shunya Ikeda ${ }^{6}$, Hideki Hashimoto ${ }^{7)}$ \\ 1) Department of Preventive Medicine and Community Health, University of Occupational and Environmental Health \\ ${ }^{2)}$ Center for Cancer Control and Information Services, National Cancer Center \\ 3) Department of Health Care Administration and Management, Graduate School of Medicine, Kyushu University \\ 4) Division of Medical Management, Hokkaido University Hospital \\ 5) Department of Health Care Informatics, Tokyo Medical and Dental University \\ 6) Department of Pharmaceutical Science, International University of Health and Welfare \\ 7) Health Economics and Epidemiology Research, School of Public Health, University of Tokyo
}

\begin{abstract}
Cancer has been the leading cause of death in Japan since 1981. In order to tackle this situation, the Cancer Control Act was approved in June 2006 and the law has been implemented since April 2007. The basic concepts of the low are 1) promotion of cancer research and utilization of research outcomes, 2) equalization of cancer medical services, and 3) development of cancer medical services to satisfy patients. In order to implement the appropriate cancer policy, the objective data of cancer treatment is indispensable. The DPC (Diagnosis Procedure Combination) scheme can contribute to the development of more appropriate and evidence based cancer policy. In this article, the authors will indicate the usefulness of DPC data in health service planning for cancer medicine, using the data of the Fukuoka Health Care Region.
\end{abstract}

Key words: cancer, health care planning, DPC

\section{Introduction}

Cancer has been the leading cause of death in Japan since 1981. The number of cancer deaths in 2006 in Japan was about 329,000. In terms of cancer sites, lung was the leading site (23\%) for males, followed by stomach $(17 \%)$, liver (11\%), colon (7\%; same rank when colon and rectum are combined: $11 \%)$, and pancreas $(6 \%)$. The leading site for females was stomach (13\%), followed by lung (13\%), colon $(10 \%)$, breast $(9 \%)$, and liver $(8 \%)$.

Considering the importance of dealing with can-

\section{Received: December 29, 2008}

Accepted: April 7, 2009

Correspondence: S. Matsuda, Department of Preventive Medicine and Community Health, University of Occupational and Environmental Health, 1-1 Iseigaoka, Yahatanishiku, Kitakyushu, Fukuoka 807-8555, Japan

e-mail: smatsuda@med.uoeh-u.ac.jp cer for the population's health, the Japanese government has implemented a series of Comprehensive 10year Strategy for Cancer Control (1 $1^{\text {st }}: 1984-1993,2^{\text {nd. }}$ 1994-2003) in order to tackle cancer. Since 2004, the $3^{\text {rd }}$-term Comprehensive 10-year Strategy for Cancer Control has been implemented. The main purpose is to promote cancer research and disseminate highquality cancer medical services. In May 2005, the Japanese Ministry of Health, Labour and Welfare (MHLW) created the Headquarters of Cancer Control in order to promote multidisciplinary activity for comprehensive cancer control, and launched the Action Plan 2005 for the promotion of Cancer Control in August.

In June 2006, the Cancer Control Act was approved and the law has been implemented since April 2007. The basic concepts of the law are 1) promotion of cancer research and utilization of research 
Table 1 Basic Plan to promote Cancer Control Programs 2007

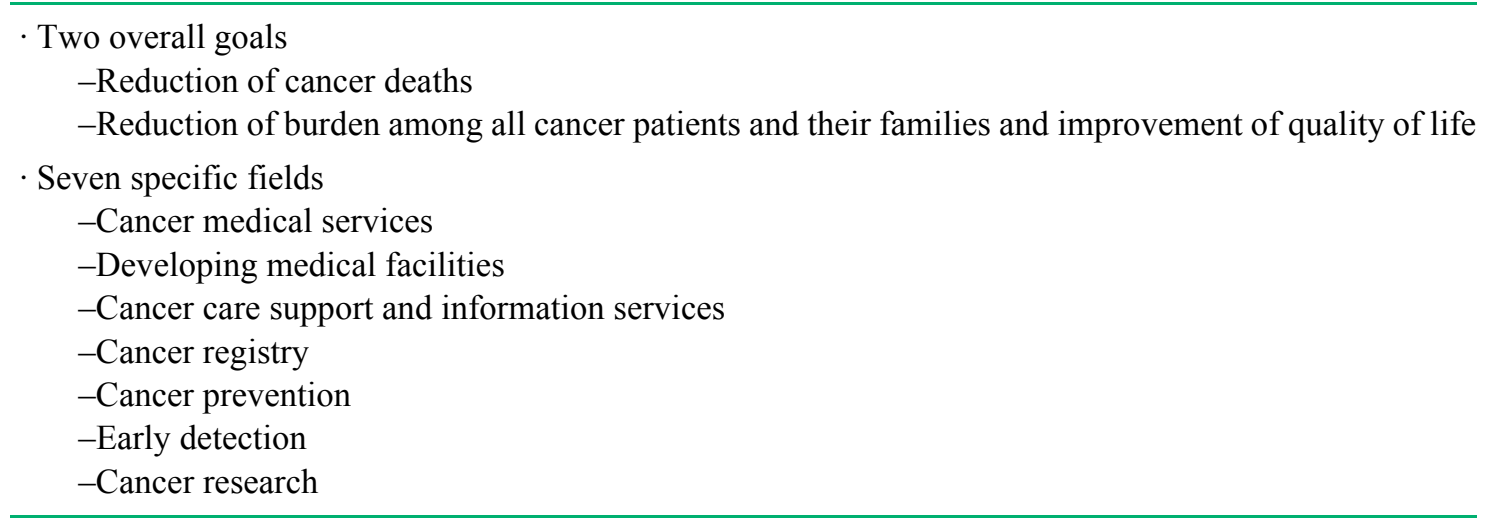

outcomes, 2) equalization of cancer medical services, and 3 ) development of cancer medical services to satisfy patients. Furthermore, according to this law, the Japanese government constructed the Basic Plan in order to promote Cancer Control Programs in June 2007, covering five fiscal years from 2007 to 2011. The Plan defines the basic concept of cancer control and aims to promote comprehensive and well-planned cancer control in Japan. According to the law, each prefecture is required to establish the Prefectural Plan to Promote Cancer Control. The Plan sets two overall goals and seven specific fields as shown in Table 1.

As cancer is the leading cause of death in Japan and the hottest issue of medical research, patients and their family, as well as the general population demand more objective and practical information about cancer treatment. It had been very difficult for the general population to know, for example, which hospital treats particular cancers most frequently, or what kinds of chemotherapy are available and popular in Japan. After the introduction of DPC (Diagnosis Procedure Combination) in 2002, the situation has drastically changed. Today, DPC data from about 1,400 acute care hospitals is available for the public. This kind of data is an important tool for development of patient-centered cancer medical services.

In this article, the authors will indicate the usefulness of DPC data in health service planning for cancer medicine.

\section{Outline of DPC classification ${ }^{1)}$}

Before describing the current study scheme, we explain the DPC concept. The basis of DPC classifi- cation is the definition table (Table 2). The first column is diagnosis that corresponds to a group of pathologies. In this case, "Malignancy, Respiratory System" contains "Malignant neoplasm of Trachea (C33)", "Malignant neoplasm of bronchus and lung (C34\$)", "Secondary malignant neoplasm of lung (C780)", "Carcinoma in situ of trachea (D021)", "Carcinoma in situ of bronchus and lung (D022)" and "Carcinoma in situ of respiratory system, unknown (D024)", for example. As the second step, a series of interventions that are usually applied are listed according to the opinion of specialist panels. Finally, other expected situation such as co-morbidities and complications are listed by the panel. Based on this definition table, our research team analyzed the actual data and constructed the DPC groups.

The structure of the DPC code consists of 8 parts as shown in Figure 1. Each part is defined by the corresponding part of the definition table. The first part is Major Diagnosis Category (Table 3) and the DPC serial number that corresponds to ICD10. The second indicates the type of admission (Current version does not use this information for grouping). The third is the code for age and birth weight. The fourth is the existence and types of surgical procedures. The fifth and sixth indicate the existence of additional procedures and adjuvant therapies, i.e. chemotherapy and radiotherapy. The seventh indicates the existence of comorbidity /complications. Finally, the eighth is the code for severity. Although the eight components are the prototype of the classification structure, it should be noticed that they are for profiling, and that all of the components are not necessarily used for reimbursement schedules. 


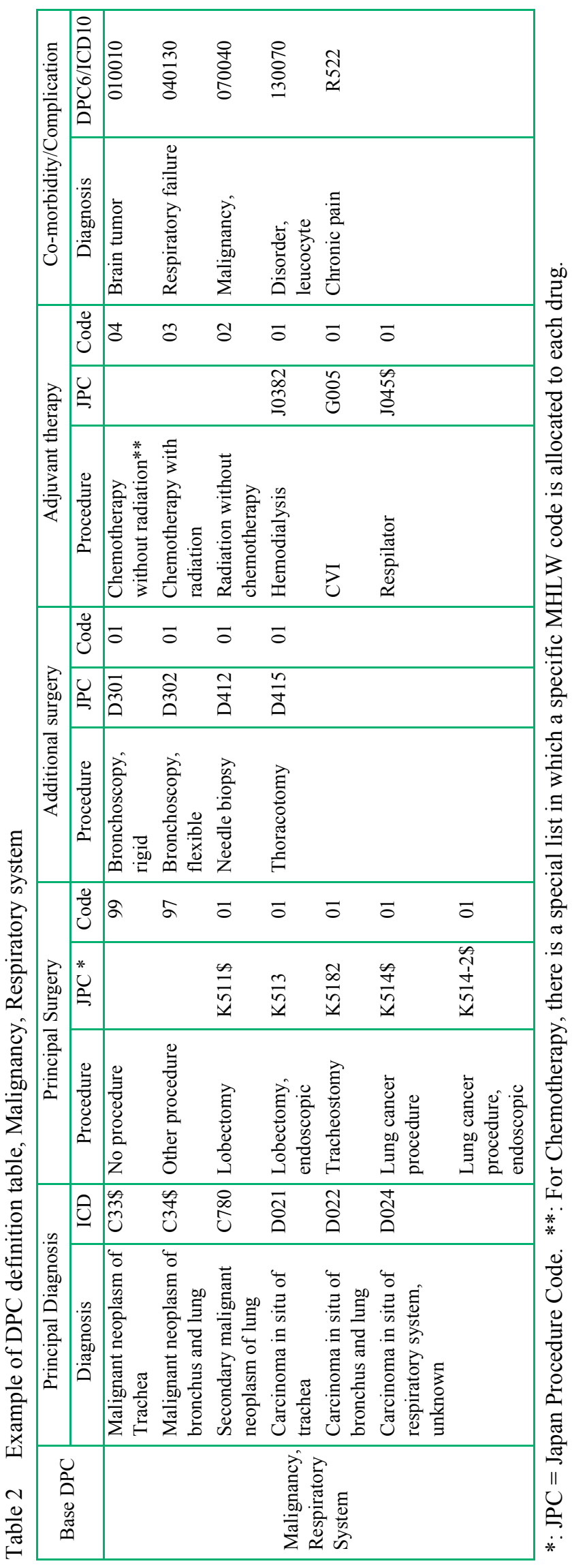




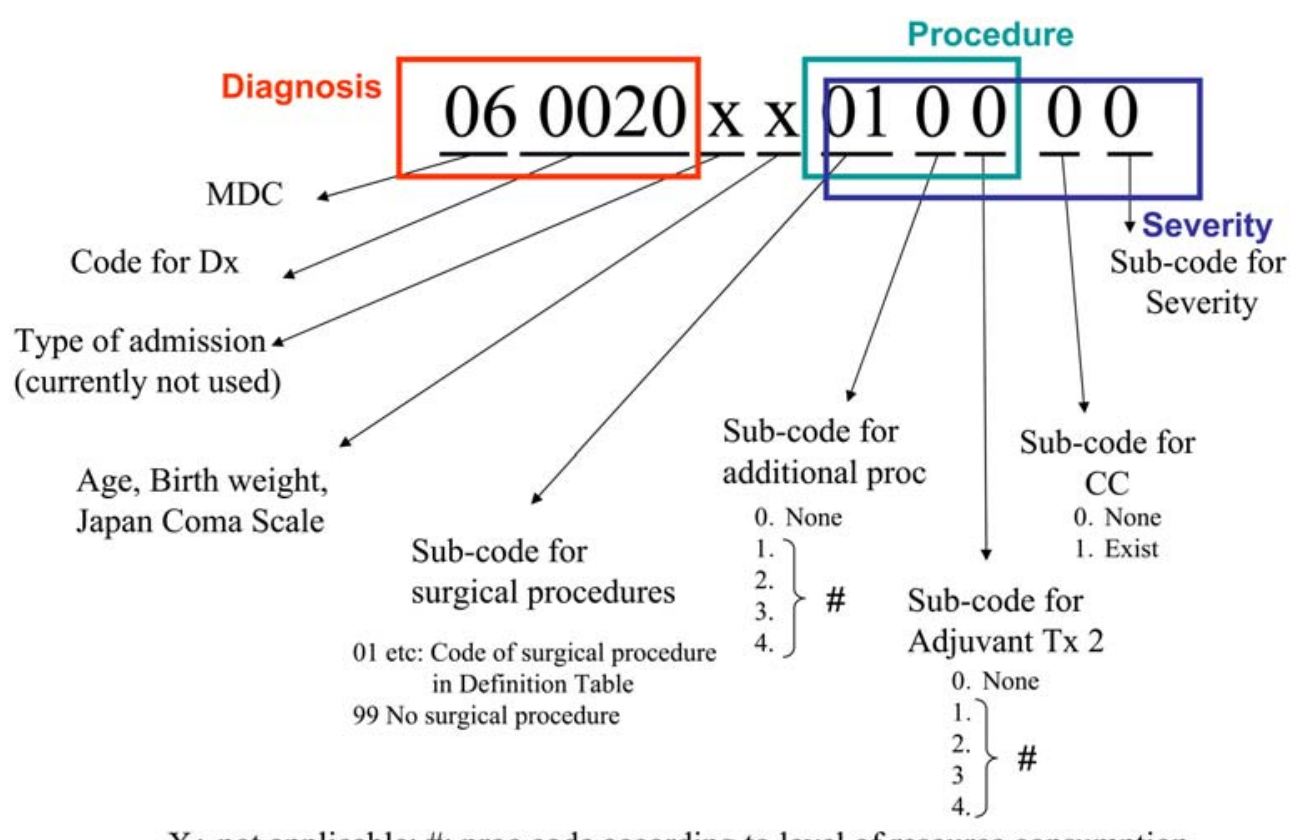

$\mathrm{X}$ : not applicable; \#: proc code according to level of resource consumption

Figure 1. Structure of code of DPC version 3.4

Table 3 Major Diagnostic Categories (2006 version)

$\begin{array}{ll}\text { MDC01 } & \text { Diseases and Disorders of the Nervous System } \\ \text { MDC02 } & \text { Diseases and Disorders of the Eye } \\ \text { MDC03 } & \text { Diseases and Disorders of the Ear, Nose, Mouth and Throat } \\ \text { MDC04 } & \text { Diseases and Disorders of the Respiratory System } \\ \text { MDC05 } & \text { Diseases and Disorders of the Circulatory System } \\ \text { MDC06 } & \text { Diseases and Disorders of the Digestive System, Hepatobiliary System and Pancreas } \\ \text { MDC07 } & \text { Diseases and Disorders of the Musculoskeletal System and Connective Tissues } \\ \text { MDC08 } & \text { Diseases and Disorders of the Skin } \\ \text { MDC09 } & \text { Diseases and Disorders of the Breast } \\ \text { MDC10 } & \text { Endocrine, Nutritional and Metabolic Diseases and Disorders } \\ \text { MDC11 } & \text { Diseases and Disorders of the Kidney, Urinary Tract and Male Reproductive System } \\ \text { MDC12 } & \text { Diseases and Disorders of the Female Reproductive System } \\ \text { MDC13 } & \text { Diseases and Disorders of the Blood, Blood Forming Organ and Myeloproliferative Diseases and Disorders } \\ \text { MCC14 } & \text { Pediatric Diseases and Disorders } \\ \text { MDC15 } & \text { Diseases and Disorders of the Newborns and Other Neonates with Conditions Originating in the Perinatal Period } \\ \text { MDC16 } & \text { Injuries, Poisonings and Toxic Effects of Drugs, Mental Diseases and Disorders and others }\end{array}$

\section{Material and Method}

The data used for this analysis was the 2007 DPC data disclosed by MHLW. This data is aggregated, not individual based. The coverage period was from July 2007 to December 2007. This dataset includes the name of the facility, the number and average length of stay for each DPC8 (the first 6 digits that indicate principal diagnosis +2 digits for main surgery) for 1,428 acute care hospitals. Another table shows the number of patients treated with chemotherapy and radio-therapy, for example. The readers must be cautious that the data table does not show the exact number for DPC8 with less than 10 cases for each hospital because of privacy issues. For convenience, we treated this case as zero in the current analysis.

As the code of main surgery is described in the definition table, we can know which hospital has done "surgical treatment of esophagus cancer with reconstruction of esophagus" the most frequently for example. 
In order to evaluate the positioning of each hospital in the health care region and in the corresponding prefecture, we prepared a table containing the names of hospitals and the number of health care regions. By combining this table with the DPC data table by MSACCESS, we constructed a dataset for the analysis.

Using this dataset, we have analyzed the actual situation of cancer treatment in the Fukuoka Health Care Region.

\section{Results}

Table 4 shows the numbers of treated cancer cases stratified by MDC and hospitals. As there are little cancer cases for MDC02, MDC05, MDC07, MDC08 and MDC10, these MDCs are excluded from the current analysis. Four hospitals; Fukuoka University Hospital, Kyushu University Hospital, Kyushu Medical Center and National Kyushu Cancer Center, are playing a principal role in cancer treatment in the Fukuoka Health Care Region. However, there are some hospitals that treat relatively many cases for specific fields; i.e., Hara Sanshin Hospital for MDC11, Hamanomachi Hospital for MDC13, Fukuoka Wajiro Hospital for MDC01, Saiseikai Fukuoka General Hospital for surgical case of MDC12, and Hakuaikai Hospital for surgical case of MDC09.

Table 5 shows the number of patients treated with chemotherapy and radio-therapy. Again, the four hospitals; Fukuoka University Hospital, Kyushu University Hospital, Kyushu Medical Center and National Kyushu Cancer Center are the main facilities in these two interventions. Fukuoka Wajiro Hospital is another important facility for radio-therapy.

Table 6 shows the number of cancer cases of MDC06 stratified by diagnosis. Although the leading four hospitals are the same as Table 4 and Table 5 , the top hospital is different in each principal diagnosis. For cancers that need intensive resources and sophisticated skills, i.e., cancer of esophagus, liver, and pancreas, Kyushu University Hospital is the leading facility. Compared with medical cases, surgical cases were more concentrated in the leading four facilities.

\section{* Discussion}

Due to the emerging consumerism, today's patients demand more information about hospital services for patient's choice. Before the introduction of the DPC scheme, there was no systematic information with which the patient could know the clinical characteristics of each hospital. For example, a patient with breast cancer experienced difficulty in obtaining objective information about which hospital treated this pathology the most in the region. Usually the patient was referred to a specialist hospital by the first contact doctor. It was very rare that the patients chose the hospital by themselves. As shown in the current study, the DPC open data is a very important source for patient's choice.

The DPC data can be used for regional hospital planning. Since 1984, the Japanese government has introduced the Regional Health Care Plan (RHCP) that regulates the number of hospital beds in the corresponding region ${ }^{2)}$. One of the most important objectives of the RHCP is to realize appropriate resource allocation within the region. This requires functional differentiation, coordination and networking among the facilities. In order to establish a workable RHCP, appropriate information about the disease structure and the performance of each facility in the region is indispensable. The reality has been quite far from the objectives of RHCP. Most of the hospitals have constructed their function without enough information about the local needs and coordination among the facilities. As a result, there are tremendous amount of duplication for the functions among the facilities. As OECD data indicates (Table 7), there are too many CT and MRI scanners in Japan ${ }^{3)}$.

The DPC framework has a possibility to drastically ameliorate the situation. The DPC research gathers very detailed data so called Form 1, Form 3, E files and $\mathrm{F}$ file. Form 1 is a patient summary that contains the following patient information; data ID number, age, sex, major diagnosis (ICD-10), co-mobility and complication (ICD-10), surgical intervention (Japanese payment code), other major procedures (Japanese payment code), emergency case or not, and outcome. Form 3 contains the data about medical resources of each hospital; i.e., number of beds, human resources (physician, nurse, OT, PT, pharmacist, etc), and medical devices (CT, MRI, PET CT, and other expensive devices). E-file has information of the bundled charge of procedure. F-file indicates the detail of bundled procedures. Form 1, E-file and F-file are matched according to the data ID number that is unique for each discharged case. By combining these data, we can describe the total process of in-patient 


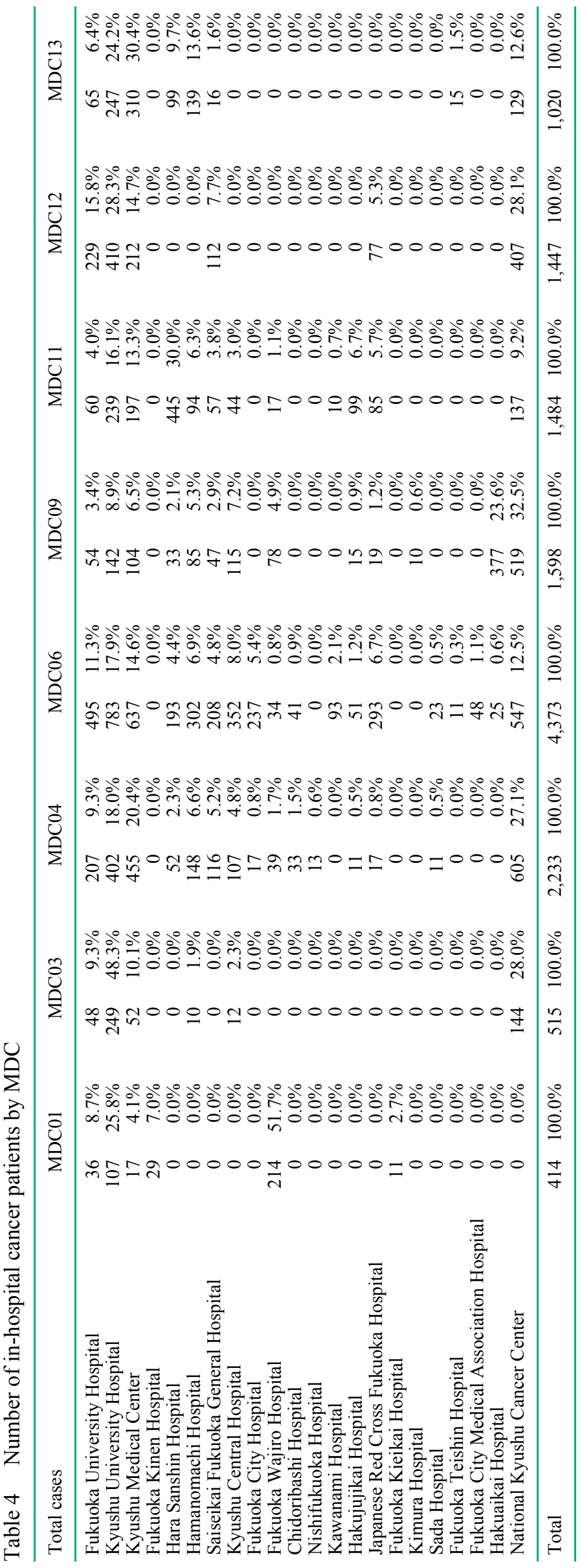

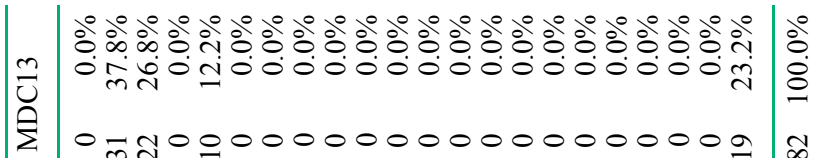

ป

$\sum$

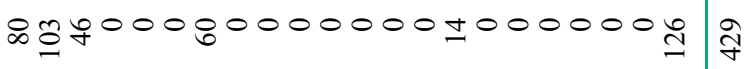

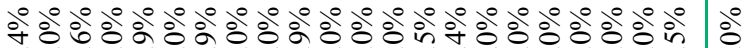
च nू⿻

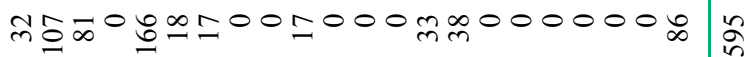

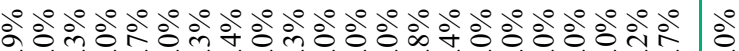

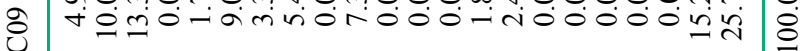
主

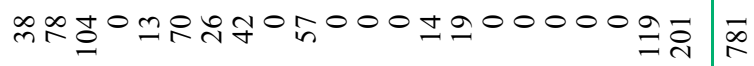

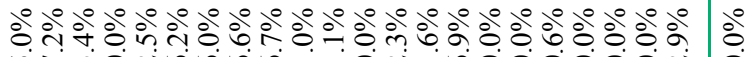

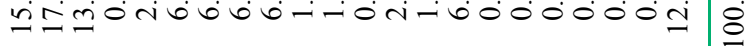
哭字示 离 ซํัลे

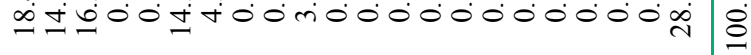

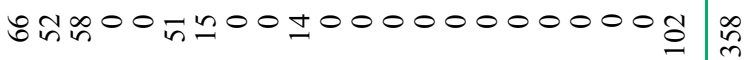

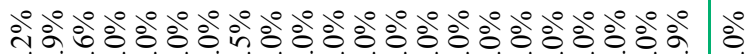

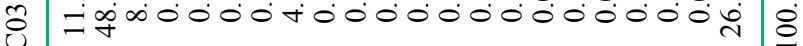
$\sum$

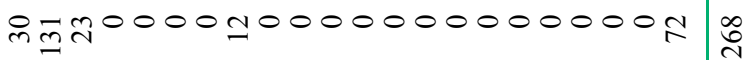

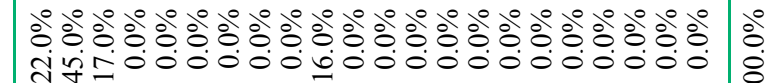
ঠृ สุน

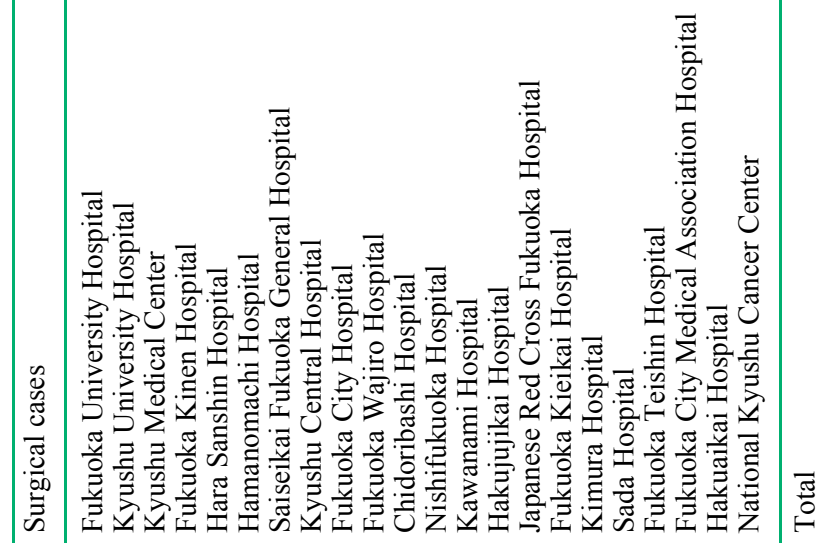


Table 5 Number of in-hospital cancer patients with chemotherapy and radio-therapyby MDC

\begin{tabular}{|c|c|c|c|c|}
\hline \multirow{2}{*}{$\begin{array}{l}\text { Total cases } \\
\text { Fukuoka University Hospital }\end{array}$} & \multicolumn{2}{|c|}{ Chemotherapy } & \multicolumn{2}{|c|}{ Radio-therapy } \\
\hline & 766 & $9.7 \%$ & 167 & $10.6 \%$ \\
\hline Kyushu University Hospital & 1,483 & $18.8 \%$ & 508 & $32.1 \%$ \\
\hline Kyushu Medical Center & 1,156 & $14.7 \%$ & 150 & $9.5 \%$ \\
\hline Fukuoka Kinen Hospital & 17 & $0.2 \%$ & 35 & $2.2 \%$ \\
\hline Hara Sanshin Hospital & 466 & $5.9 \%$ & 0 & $0.0 \%$ \\
\hline Hamanomachi Hospital & 433 & $5.5 \%$ & 96 & $6.1 \%$ \\
\hline Saiseikai Fukuoka General Hospital & 305 & $3.9 \%$ & 68 & $4.3 \%$ \\
\hline Kyushu Central Hospital & 382 & $4.8 \%$ & 84 & $5.3 \%$ \\
\hline Fukuoka City Hospital & 205 & $2.6 \%$ & 0 & $0.0 \%$ \\
\hline Fukuoka Wajiro Hospital & 116 & $1.5 \%$ & 193 & $12.2 \%$ \\
\hline Chidoribashi Hospital & 81 & $1.0 \%$ & 0 & $0.0 \%$ \\
\hline Nishifukuoka Hospital & 16 & $0.2 \%$ & 0 & $0.0 \%$ \\
\hline Kawanami Hospital & 71 & $0.9 \%$ & 0 & $0.0 \%$ \\
\hline Hakujujikai Hospital & 89 & $1.1 \%$ & 0 & $0.0 \%$ \\
\hline Japanese Red Cross Fukuoka Hospital & 315 & $4.0 \%$ & 30 & $1.9 \%$ \\
\hline Fukuoka Kieikai Hospital & 12 & $0.2 \%$ & 0 & $0.0 \%$ \\
\hline Kimura Hospital & 28 & $0.4 \%$ & 0 & $0.0 \%$ \\
\hline Sada Hospital & 36 & $0.5 \%$ & 0 & $0.0 \%$ \\
\hline Fukuoka Teishin Hospital & 33 & $0.4 \%$ & 0 & $0.0 \%$ \\
\hline Fukuoka City Medical Association Hospital & 0 & $0.0 \%$ & 0 & $0.0 \%$ \\
\hline Hakuaikai Hospital & 263 & $3.3 \%$ & 0 & $0.0 \%$ \\
\hline National Kyushu Cancer Center & 1,613 & $20.5 \%$ & 250 & $15.8 \%$ \\
\hline Total & 7,886 & $100.0 \%$ & 1,581 & $100.0 \%$ \\
\hline
\end{tabular}

Table 7 International comparison of health resources (2006)

\begin{tabular}{|c|c|c|c|c|c|c|c|c|c|}
\hline Nation & $\begin{array}{c}\text { Beds per } \\
1,000 \\
\text { inhabitants }\end{array}$ & $\begin{array}{c}\text { No ofDr } \\
\text { per } 100 \\
\text { beds }^{\mathrm{a}}\end{array}$ & $\begin{array}{c}\text { No of Dr } \\
\text { per } 1,000 \\
\text { inhabitants }\end{array}$ & $\begin{array}{c}\text { No of Ns } \\
\text { per } 100 \\
\text { beds }^{\mathrm{a}}\end{array}$ & $\begin{array}{c}\text { No of Ns } \\
\text { per } 1,000 \\
\text { inhabitants }\end{array}$ & $\begin{array}{c}\text { No ofCT } \\
\text { per } 1 \mathrm{M} \\
\text { inhabitants }\end{array}$ & $\begin{array}{c}\text { No of MRI } \\
\text { per } 1 \mathrm{M} \\
\text { inhabitants }\end{array}$ & $\begin{array}{c}\text { ALOS } \\
\text { acute } \\
\text { care beds }\end{array}$ & $\begin{array}{c}\text { UR } \\
\text { Out- } \\
\text { patients }^{\mathrm{a}}\end{array}$ \\
\hline Japan & 14.0 & 14.3 & 2.1 & 63.2 & 9.4 & $92.6^{\mathrm{b}}$ & $40.1^{\mathrm{c}}$ & 19.2 & 13.8 \\
\hline Germany & 8.3 & 39.5 & 3.5 & 113.0 & 9.9 & 15.8 & 7.7 & 7.9 & 7.0 \\
\hline France & 7.2 & 44.9 & 3.4 & 100.1 & 7.9 & 10.0 & 5.3 & 5.4 & 6.6 \\
\hline UK & 3.6 & 57.5 & 2.4 & 227.7 & 10.0 & 7.6 & 5.6 & 7.5 & 5.3 \\
\hline USA & 3.2 & 73.3 & 2.4 & 237.9 & 10.6 & 34.0 & 26.5 & 5.5 & 3.8 \\
\hline
\end{tabular}

a; 2004, ${ }^{\text {b; } 2002, ~}{ }^{\text {c }} ; 2005$.

Source: OECD Health Data 2008 (2008)

services and standard CT or MRI utilization frequency for each DPC. Using this information we will be able to estimate the appropriate numbers of CT scanners and MRI scanners for each health care region and promote the co-utilization of such devices.

As shown in Table 7, Japan has too many general beds compared with other developed countries ${ }^{3)}$. This over-bed situation has been managed by smaller number of health professionals compared with other countries. It is considered that this situation is one of the reasons of recent burn-out phenomenon among the health professionals of acute-care hospital. It is not rare for a 40-yr-old physician to work more than $60 \mathrm{~h}$ a week. Differentiation, regionalization and coordination of hospital function within the same region will make it possible for better working conditions for health professionals and assuring clinical safety. This is very important for acute care services such as cancer, cardiovascular diseases, and emergency.

In the case of cancer treatment within the Fukuoka Health Care Region, as the current study has indicated, the leading four hospitals treat more than $70 \%$ 


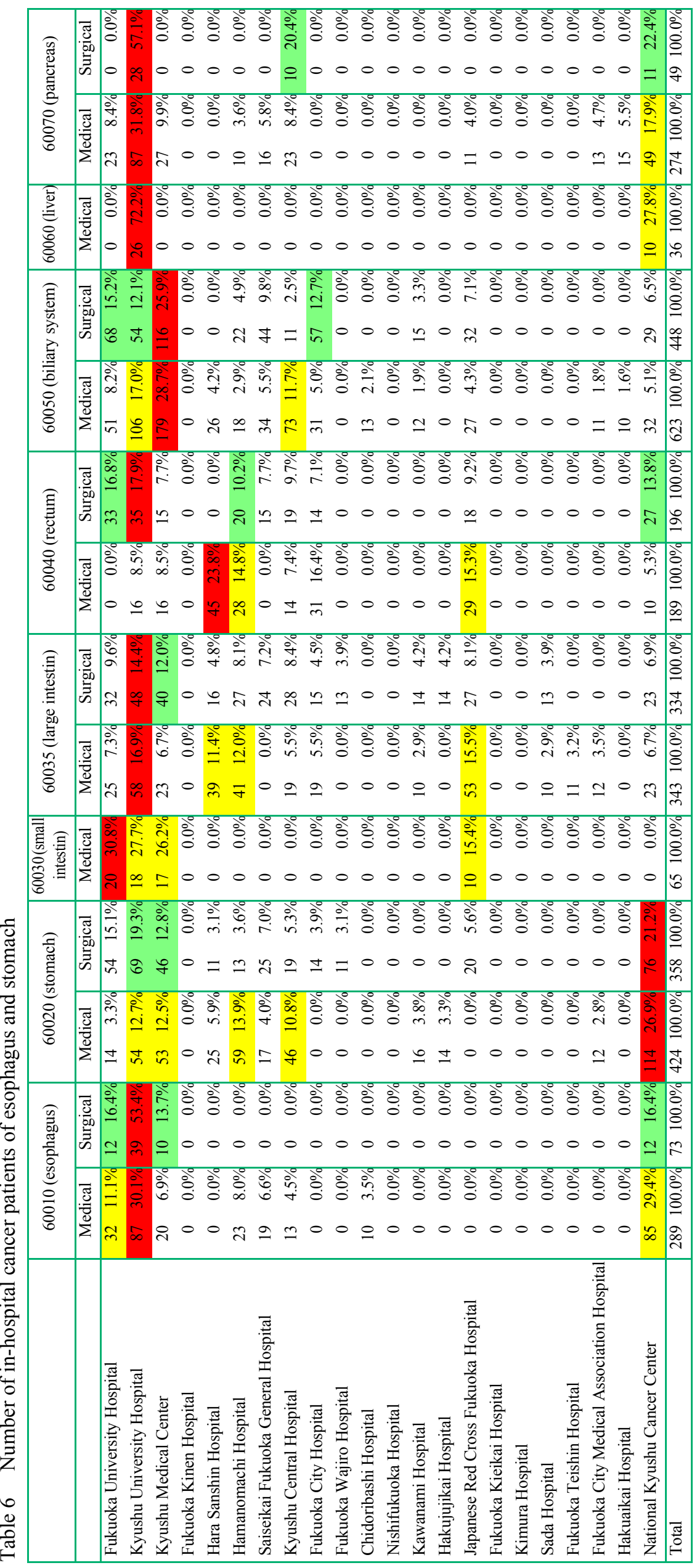


of total cancer patients and $80 \%$ of surgical cases. Another 20 to $30 \%$ of patients were treated in the remaining 18 facilities. This situation can be evaluated that the functional differentiation is much advanced in the region compared with other health care regions. However, the level of coordination and networking cannot be evaluated from the current data.

Recently the necessity of Evidence Based Health Policy Making (EBHPM) has been discussed under the New Public Management movement. We must be very cautious that policy makers tend to prefer simple messages and interventions more under their control. If the volume-quality relationship hypothesis is true, the DPC based volume data will become an important tool for rational hospital planning. That is, policy makers can request the merge and closure of specific departments of hospital in order to assure the quality of medical services. Considering the current situation of over-equipment of beds and medical devices in Japan, this scenario will be very attractive for policy makers. In fact, research since the late 1970s seemed to point in the direction of a relatively constant relationship in health care, that patients treated in hospitals which managed high volumes of patients with the same condition had better outcomes than those with lower volumes ${ }^{4}$.

However, another well controlled study did not show a strong association between volume and quality ${ }^{5}$. More importantly, the policy of concentrating services may result in reduced local access for services. In the case of cancer treatment, it seems to be more appropriate to assure accessibility for screening and the following detailed examination even though concentration of specific functions like surgical procedures and radio-therapy may be preferable for quality assurance and management efficiency. In this perspective, the situation of Fukuoka Health Care Region might be acceptable. In this region, non-surgical procedures such as cancer screening and medical treatment are offered by many facilities and the surgical procedures are mainly done by the leading four hospitals.

As this study has indicated, the DPC open data is very useful for hospital profiling and is a powerful tool for regional health care service planning. However, there are several limitations at the current format. First, the current DPC study covers only 1,428 hospitals among the 9,000 hospitals. Although 1,428 hospitals are the leading acute care hospitals covering more than $80 \%$ of acute phase in-patients, the cover- age must be improved. As Fushimi and Matsuda suggested, the DPC logic can be applied for chronic care hospital and out-patient services ${ }^{6}$. In order to describe the disease structure more precisely for health policy making, the electronization of the whole range of medical services is absolutely necessary. Second, the tables did not show the DPC with less than 10 cases because of privacy issue. Considering the Japanese situation where many hospitals receive relatively small number of particular DPCs, estimated disease structures might be underestimated and skewed for high-volume hospitals. Third, the current data covers only 6 months. Thus we cannot evaluate the effect of seasonal fluctuation. The data collection must be 12 months in length. Fourth, disclosed information is too rough to evaluate the clinical level of each hospital. More detailed data, such as more detailed information about intervention, must be included into the open data. Now MHLW is preparing a framework of the DPC database that will be disclosed for the public. As Fushimi and Matsuda indicated $^{6}$, the application of the DPC logic to Patient Survey will be useful for health policy making. It is expected that the DPC framework will be used in health policy making in the future.

\section{References}

1) Matsuda S: Diagnosis Procedure Combination - the Japanese original casemix system-, In: Kimberly J and Pouvourville G (ed), the Globalization of Managerial Innovation in Health Care, Cambridge University Press, 2008.

2) Matsuda S: Re-organization of the Japanese hospital system. Journal of Economics and Medicine 22, 6978 (2004).

3) OECD: OECD Health data 2008, Paris: OECD, 2008.

4) Luft HS, Garnick DW, Mark DH, et al.: Hospital volume, physician volume and patient outcomes. Assessing evidence. Michigan: Health Administration Press.

5) Sheikh K. Reliability of provider volume and outcome associations healthcare policy, Med Care 41, 1111-1117 (2003).

6) Fushimi K and Matsuda S: Application of Diagnosis Procedure Combination Case Mix system and National Patient Database to Regional Estimation of Disease Structure, APJDM 1, 29-33 (2007). 